\title{
5-Fluorouracil/irinotecan induced lethal toxicity as a result of a combined pharmacogenetic syndrome: report of a case
}

\author{
M Steiner, M Seule, B Steiner, I Bauer, M Freund, C H Köhne, P Schuff-Werner
}

J Clin Pathol 2005;58:553-555. doi: 10.1136/icp.2004.022319

Combination cancer chemotherapy induced toxicity can be associated with combined pharmacogenetic syndromes. Dihydropyrimidine dehydrogenase (DPD) is the principal enzyme involved in the catabolic detoxification of 5fluorouracil (5FU). A heterozygous $G>A$ transition at the $5^{\prime}$ splicing donor consensus sequence in intron 14 leading to exon 14 skipping (IVS14+1 G > A, DPYD*2A) with partial loss of enzyme activity may be partly responsible for $5 \mathrm{FU}$ induced toxicity, whereas irinotecan associated toxicity may in part be explained by an aberrant UGT1A1 promoter (TA $)_{n}$ genotype underlying Gilbert's syndrome with reduced liver glucuronidation activity. This report describes a 44 year old white woman who suffered from severe gastrointestinal and haematological toxicity while undergoing $5 \mathrm{FU}_{24 \mathrm{~h}} /$ folinic acid/irinotecan treatment for adenocarcinoma of the sigmoid colon. Despite appropriate supportive treatment, her condition rapidly deteriorated and led to death. Molecular analysis revealed a hitherto undescribed combined pharmacogenetic syndrome, consisting of heterozygosity for the DPD IVS14+1 G > A mutation and UGTIA1 (TA) $6 / 7$ heterozygosity, which probably contributed to the fatal outcome in this patient.

$\mathrm{D}$ ihydropyrimidine dehydrogenase (DPD, EC 1.3.1.2.) is the first and rate limiting enzyme of the pyrimidine catabolic pathway, with more than $80 \%$ of an administered dose of the chemotherapeutic drug 5-fluorouracil (5FU) being degraded by the enzyme, thus reducing its efficiency as a cytotoxic drug, and reducing drug toxicity due to accumulation of toxic metabolites. Following the initial description of familial pyrimidinaemia and severe neurotoxicity after 5FU treatment, ${ }^{1}$ several reports appeared supporting the association between partial or complete DPD deficiency and severe 5FU induced toxicity, including lethal outcomes. ${ }^{2}$ Detailed investigations of the molecular genetics of DPD deficiency identified approximately 40 different genetic aberrations, including exon skipping, deletions, frameshifts, missense mutations, and polymorphisms.

\section{"Several reports support the association between partial or complete dihydropyrimidine dehydrogenase deficiency and severe 5-fluorouracil induced toxicity, including lethal outcomes"}

In white populations, it is generally accepted that a heterozygous $5^{\prime}$ splice site mutation in intron 14 (DPYD IVS14+1 G > A, designated DPYD*2A) occurs with a frequency of $1-2 \%$ in the general population..$^{3-7}$ This mutation leads to exon 14 skipping and an altered protein, with reduced enzyme activity in heterozygotes and complete lack of catalytic activity in homozygotes.
The topoisomerase I inhibitor irinotecan displays potent activity against gastrointestinal malignancies and is being used in combined cancer chemotherapy approaches. ${ }^{8}$ The presence of reduced liver glucuronidation activity as a result of an aberrant UGTlAl promoter (TA) n genotype, which underlies Gilbert's syndrome, contributes to irinotecan toxicity. ${ }^{9-11}$

We report a hitherto undescribed combined pharmacogenetic syndrome consisting of heterozygosity for DPYD*2A in the presence of a heterozygous UGTlAl promoter (TA) 6/7 genotype underlying Gilbert's syndrome causing lethal $5 \mathrm{FU}_{24 \mathrm{~h}}$ /folinic acid (FA)/irinotecan induced toxicity in a patient suffering from adenocarcinoma of the sigmoid colon.

\section{CASE REPORT}

A 44 year old white, female index patient (BS) was diagnosed with a moderately differentiated adenocarcinoma of the sigmoid colon and underwent hemicolectomy and lymphadenectomy in 1995. As a result of liver metastases, hemihepatectomy was performed four years later. An exploratory laparotomy performed in January 2001 revealed advanced metastatic disease. Combined chemotherapy according to EORTC protocol 40986 was initiated and consisted of irinotecan $\left(80 \mathrm{mg} / \mathrm{m}^{2}, 30\right.$ minute infusion), 5FU (2000 mg/m², 24 hour infusion), and FA $\left(500 \mathrm{mg} / \mathrm{m}^{2}\right.$, two hour infusion) on a weekly basis. Nausea and vomiting (CTC grade 2), leucopenia (grade 1), and severe fatigue necessitated dose reduction after the second administration (irinotecan $64 \mathrm{mg} / \mathrm{m}^{2}$, 5FU $1600 \mathrm{mg} / \mathrm{m}^{2}$ ). After dose reduction, no immediate nausea and vomiting were noted. However, the clinical condition deteriorated progressively to grade 4 toxicity (diarrhoea, neutropenia) and was further complicated by sepsis. Despite intensive medical care, the patient died from multiorgan failure.

DPYD*2A mutation genotyping by polymerase chain reaction mediated site directed mutagenesis followed by restriction fragment length polymorphism with the SnabI restriction enzyme ${ }^{12}$ identified heterozygosity (fig 1), which was independently confirmed using a polymerase chain reaction restriction fragment length polymorphism assay with MaeII digestion. ${ }^{3}$ In addition, UGTIAl promoter analysis was performed, ${ }^{13}$ and demonstrated a heterozygous UGTIAl (TA) $6 / 7$ genotype underlying Gilbert's syndrome (fig 2).

\section{DISCUSSION}

Partial or complete deficiency of the rate limiting enzyme in pyrimidine catabolism, DPD, is increasingly being considered as a contributing factor to the occurrence and severity of $5 \mathrm{FU}$ toxicity. However, the overall impact of this pharmacogenetic syndrome on adverse drug reactions to treatment with fluoropyrimidine has not been established conclusively.

Abbreviations: DPD, dihydropyrimidine dehydrogenase; FA, folinic acid; 5FU, 5-fluorouracil 


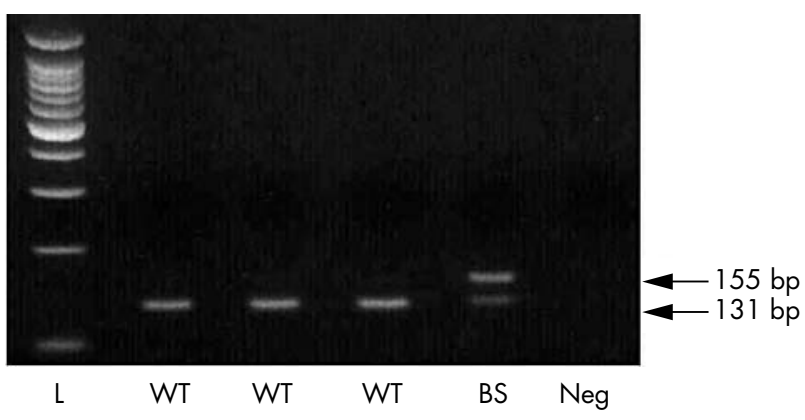

Figure 1 Polymerase chain reaction (PCR) mediated site directed mutagenesis followed by restriction fragment length polymorphism assay to detect DPYD IVS14+1 G > A (DPYD*2A). After digesting the PCR product of 155 bp length with Snabl, two ethidium bromide stained bands of 155 and 131 bp length were seen for the patient (BS), indicating heterozygosity for DPYD*2A. Homozygosity for the wild-type DPYD allele with a single 131 bp band is shown for comparison (WT), in addition to a negative control (Neg) and a DNA ladder (L).

Severe 5FU induced toxicity including a lethal outcome has been reported in several heterozygous DPYD ${ }^{\star} 2 \mathrm{~A}$ mutation carriers. In addition, fatal fluoropyrimidine induced toxicity in patients with complete DPD deficiency and underlying homozygous DPYD*2A mutation has been reported. ${ }^{26714}$

Treatment for advanced colorectal cancer with $5 \mathrm{FU}_{24 \mathrm{~h}} / \mathrm{FA} /$ irinotecan according to EORTC protocol 40986 in a 44 year old female patient who subsequently proved to be heterozygous for the DPYD*2A mutation caused severe toxicity with a lethal outcome, thus supporting the concept that mutation carriers are at increased risk for fluoropyrimidine induced life threatening toxicity. However, several points need to be considered in the case presented here. First, a thorough phenotypic investigation was unfortunately not available for the patient. Therefore, the patient's residual DPD enzyme activity remains speculative. Second, the index patient underwent combined palliative chemotherapy with $5 \mathrm{FU}_{24 \mathrm{~h}}$, FA, and the topoisomerase I inhibitor irinotecan. Irinotecan itself has been implicated in a pharmacogenetic syndrome causing substantial drug induced toxicity. ${ }^{15}$ The prodrug irinotecan is bioactivated to the active compound SN-38, which in turn is the substrate for inactivating metabolic pathways consisting predominantly of glucuronide conjugation catalysed by liver microsomal uridine diphosphate glucurunosyltransferase, UGTIAl. Allelic $(\mathrm{TA})_{\mathrm{n}}$ variants in the UGTIAl promoter region, as seen in mild hereditary unconjugated hyperbilirubinaemia syndromes, are associated with reduced enzyme activity, which by impaired SN-38 conjugation could lead to toxicity. Indeed, severe irinotecan induced toxicity has been reported in two patients with metastatic colon cancer and hereditary unconjugated hyperbilirubinaemia or Gilbert's syndrome. ${ }^{9}$ Pharmacogenetic analysis revealed that aberrant UGTIAl promoter alleles represent a significant risk factor for severe irinotecan toxicity. ${ }^{10}$ Recent studies indicated that grade 4 neutropenia occurred much more frequently in patients with homozygous or heterozygous Gilbert's syndrome than in wild-type carriers when irinotecan was administered as single drug treatment. ${ }^{11}$

"The coexistence of more than one pharmacogenetic syndrome in a single patient seems possible"

Careful retrospective analysis of our index patient's laboratory data revealed that pathological bilirubin concentrations were not noted, except slightly increased values immediately after abdominal surgery, which is not

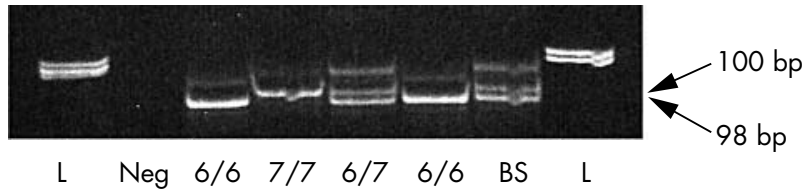

Figure 2 Polymerase chain reaction (PCR) based UGT1A1 promoter $(T A)_{n}$ genotyping assay. PCR amplification of genomic DNA was performed using specific primers and the products were resolved on a polyacrylamide gel. Ethidium bromide stained 98 and 100 bp bands correspond to six and seven UGT1A1 promoter TA repeats, respectively. Heterozygosity for UGT1A1 TA $6 / 7$ was demonstrated for the index patient (BS). Samples with known genotype, a negative control (Neg), and DNA ladders (110 and $111 \mathrm{bp}$ bands; L) were included for comparison.

considered indicative of a hereditary unconjugated hyperbilirubinaemia. Nevertheless, UGTIAl promoter analysis revealed the UGTIAl (TA) 6/7 genotype in our index patient. It has been estimated that $(\mathrm{TA})_{6 / 7}$ heterozygotes have a $25 \%$ decrease in SN-38 glucuronidation activity, ${ }^{16}$ and either heterozygosity or homozygosity for the UGTIAl (TA) 7 allele confers a high risk (odds ratio, 7.2; 95\% confidence interval 2.5 to 22.3 ) for severe irinotecan toxicity. ${ }^{10}$ Therefore, the aberrant UGTIAl promoter genotype probably contributed to the severe toxicity seen in our index patient while undergoing combined treatment with $5 \mathrm{FU}_{24 \mathrm{~h}} / \mathrm{FA} /$ irinotecan. This clearly illustrates that the coexistence of more than one pharmacogenetic syndrome in a single patient seems possible. Combined pharmacogenetic syndromes should be carefully investigated in patients with cancer showing severe toxicity while receiving several different chemotherapeutic agents.

Predictive pharmacogenetic testing is considered a potentially powerful approach to individualise cancer chemotherapy. With regard to fluoropyrimidine associated adverse drug reactions, currently available data are limited, rendering a recommendation for DPYD gene mutation(s) screening premature. In contrast, the association between Gilbert's syndrome and irinotecan toxicity is firmly established, calling for pretherapeutic testing to reduce high grade toxicity. ${ }^{17}$

In conclusion, the impact of a hitherto undescribed combined pharmacogenetic syndrome consisting of heterozygosity for DPYD*2A and UGTIAl $(\mathrm{TA})_{6 / 7}$ as related to $5 \mathrm{FU}_{24 \mathrm{~h}} / \mathrm{FA} /$ irinotecan induced severe toxicity was demonstrated, thus calling for combined pharmacogenetic approaches to combined cancer chemotherapy associated adverse drug reactions.

Take home messages

- We describe a patient who suffered severe gastrointestinal and haematological toxicity while undergoing combination 24 hour 5-fluorouracil/folinic acid/irinotecan treatment for adenocarcinoma of the sigmoid colon, which led to her death

- Molecular analysis revealed a novel combined pharmacogenetic syndrome, consisting of heterozygosity for the dihydropyrimidine dehydrogenase IVS14+1 $\mathrm{G}>\mathrm{A}$ mutation and UGT1A1 (TA) $6 / 7$ heterozygosity, which resulted in 5-fluorouracil/irinotecan intolerance and probably contributed to the fatal outcome

- Combined pharmacogenetic approaches are required for adverse drug reactions associated with combined cancer chemotherapy 


\section{Authors' affiliations}

M Steiner, M Seule, P Schuff-Werner, Institute of Clinical Chemistry and Laboratory Medicine, University of Rostock, Ernst-Heydemann-Str. 6, D18057 Rostock, Germany

B Steiner, M Freund, C H Köhne, Division of Haematology/Oncology, Department of Internal Medicine, University of Rostock

I Baver, Division of Medical Genetics, Children's Hospital, University of Rostock

Full permission was given for this case report to be published.

Correspondence to: $\operatorname{Dr} M$ Steiner, Medical Faculty, Institute of Clinical Chemistry and Laboratory Medicine, University of Rostock, ErnstHeydemann-Str. 6, D-18057 Rostock, Germany; michael.steiner@med. uni-rostock.de

Accepted for publication 22 September 2004

\section{REFERENCES}

1 Tuchman M, Stoeckeler JS, Kiang DT, et al. Familial pyrimidinemia and pyrimidinuria associated with severe fluorouracil toxicity. N Engl I Med 1985;313:245-9.

2 van Kuilenburg AB. Dihydropyrimidine dehydrogenase and the efficacy and toxicity of 5-fluorouracil. Eur J Cancer 2004;40:939-50.

3 Wei X, McLeod HL, McMurrough J, et al. Molecular basis of human dihydropyrimidine dehydrogenase deficiency and 5-fluorouracil toxicity. J Clin Invest 1996;98:610-15.

4 Wei $X$, Elizondo G, Sapone A, et al. Characterization of the human dihydropyrimidine dehydrogenase gene. Genomics 1998;51:391-400.

5 Nauck M, Gierens H, März W, et al. Rapid detection of a common dihydropyrimidine dehydrogenase mutation associated with 5 -fluorouracil toxicity and congenital thymine uraciluria using fluorogenic hybridization probes. Clin Biochem 2001;34:103-5.

6 Raida M, Schwabe W, Häusler P, et al. Prevalence of a common point mutation in the dihydropyrimidine dehydrogenase (DPD) gene within the 5 - splice donor site of intron 14 in patients with severe 5 -fluorouracil (5-FU)-related toxicity compared with controls. Clin Cancer Res 2001;7:2832-9.

7 van Kuilenburg AB, Muller EW, Haasjes J, et al. Lethal outcome of a patient with a complete dihydropyrimidine dehydrogenase (DPD) deficiency after administration of 5-fluorouracil: frequency of the common IVS14+1G >A mutation causing DPD deficiency. Clin Cancer Res 2001;7:1149-53.

8 Douillard JY, Cunningham D, Roth AD, et al. Irinotecan combined with fluorouracil compared with fluorouracil alone as first-line treatment for metastatic colorectal cancer: a multicentre randomised trial. Lancet 2000;355:1041-7.

9 Wasserman E, Myara A, Lokiec F, et al. Severe CPT-11 toxicity in patients with Gillbert's syndrome: two case reports. Ann Oncol 1997:8:1049-51.

10 Ando $Y$, Saka H, Ando M, et al. Polymorphisms of UDPglucuronosyltransferase gene and irinotecan toxicity: a pharmacogenetic analysis. Cancer Res 2000;60:6921-6.

11 Innocenti F, Undevia SD, lyer L, et al. Genetic variants in the UDP. glucuronosyltransferase $1 \mathrm{~A} 1$ gene predict the risk of severe neutropenia of irinotecan. J Clin Oncol 2004;22:1382-8.

12 Jézéquel $P$, Joalland MP, Milano G, et al. Common DPYD mutation associated with 5-fluorouracil toxicity detected by PCR-mediated site-directed mutagenesis. Clin Chem 2000;46:309-10.

13 Beutler E, Gelbart T, Demina A. Racial variability in the UDPglucuronosyltransferase 1 (UGTIA 1) promoter: a balanced polymorphism for regulation of bilirubin metabolism? Proc Natl Acad Sci U S A 1998:95:8170-4

14 van Kuilenburg AB, Meinsma JR, Zoetekouw L, et al. High prevalence of the IVS14+1 G>A mutation in the dihydropyrimidine dehydrogenase gene of patients with severe 5 -fluoruracil-associated toxicity. Pharmacogenetics 2002;12:555-8.

15 Innocenti F, lyer L, Ratain MJ. Pharmacogenetics of anticancer agents: lessons from amonafide and irinotecan. Drug Metab Dispos 2001;29:596-600.

16 lyer L, Hall D, Das S, et al. Phenotype-genotype correlation of in vitro SN-38 (active metabolite of irinotecan) and bilirubin glucuronidation in human liver tissue with UGT1A1 promoter polymorphism. Clin Pharmacol Ther 1999;65:576-82.

17 McLeod HL, Watters JW. Irinotecan pharmacogenetics: is it time to intervene? $J$ Clin Oncol 2004;22:1356-9. 